\title{
Correction to: Bilobalide Induces Neuronal Differentiation of P19 Embryonic Carcinoma Cells via Activating Wnt/ $\beta$-Catenin Pathway
}

\author{
Mei Liu' ${ }^{1}$ Jingjing Guo ${ }^{1}$ Juan Wang ${ }^{1} \cdot$ Luyong Zhang $^{1,2} \cdot$ Tao Pang $^{1,2} \cdot$ Hong Liao $^{1,2}$
}

Published online: 6 November 2019

(c) Springer Science+Business Media, LLC, part of Springer Nature 2019

\section{Correction to: \\ Cellular and Molecular Neurobiology (2014) 34:913-923 https://doi.org/10.1007/s10571-014-0072-7}

The original version of this article unfortunately contained an error in Figures $1 \mathrm{~A}$ and $4 \mathrm{C}$.

In Fig. 1A, staining picture of DMSO group was given incorrectly the same as of the image in DMSO group in Fig. 2A, and image in Bilobalide $(0.2 \mu \mathrm{m})$ was given mistakenly the same as of the image Bilobalide (2 days) in Fig. 2A.

In Fig. 4C immunostaining picture in DMSO was given inadvertently the same as of the image XAV939+Bilobalide.

Hence, the correct Figs. 1A and 4C was given below:

The original article can be found online at https://doi.org/10.1007/ s10571-014-0072-7.

Tao Pang

tpang@cpu.edu.cn

$\triangle$ Hong Liao

liaohong56@hotmail.com

1 Neurobiology Laboratory, National Center for Drug

Screening, China Pharmaceutical University,

\#24 Tongjiaxiang Street, Nanjing 210009,

People's Republic of China

2 Key Laboratory of Drug Quality Control and Pharmacovigilance (China Pharmaceutical University), Ministry of Education, Nanjing 210009, People's Republic of China 
Fig. 1 The effect of bilobalide on P19 cells differentiation in a concentration-dependent manner. a Immunofluorescence staining was performed for detecting P19 EC cells differentiation. $\beta$ III-tubulin (1:300) was used as neuron marker. Scale bar $50 \mu \mathrm{m}$. b P19 cells differentiation was determined by Western blot. $\beta$-bactin was used as endogenous control. Quantitative assessment of Western blot was shown below.

Values were reported as mean $\pm \mathrm{SD} . * P<0.05, * * P<0.01$ versus DMSO group

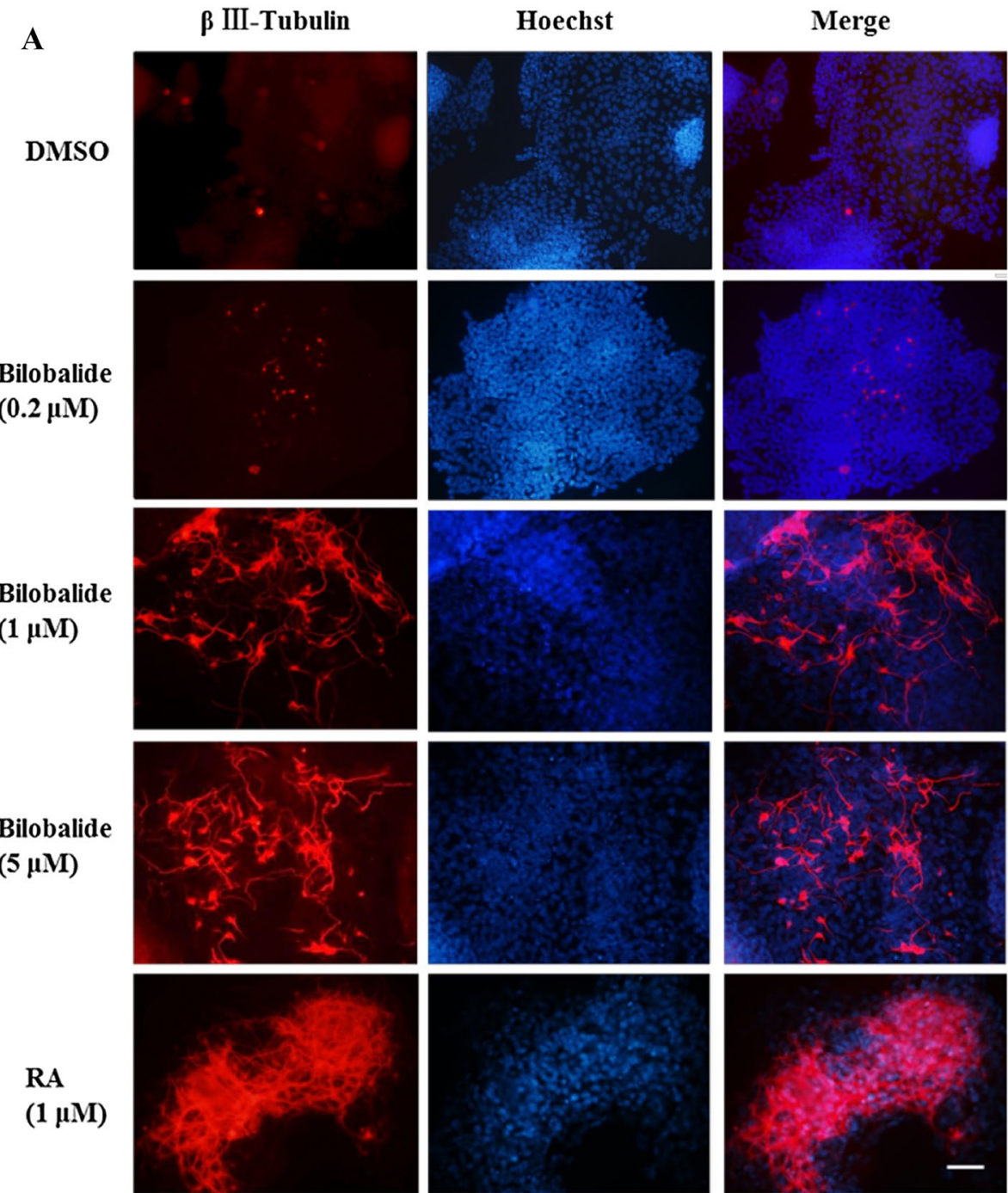


Fig. 4 Inhibitory effect of

XAV939 on bilobalide-induced

neuronal differentiation of P19

cells. Cells were pretreated with

or without $1 \mu \mathrm{mol} / \mathrm{L} \mathrm{XAV939}$

for $30 \mathrm{~min}$ before the addition

of bilobalide $(1 \mu \mathrm{mol} / \mathrm{L})$. a, b

Western blotting confirmed that

$\beta$-catenin and $\beta$ III-tubulin levels were effectively down-regulated in XAV939-treated P19 cells. c

Immunofluorescence staining with $\beta$-catenin (Red) was used to assess changes in nuclear and cytoplasmic $\beta$-catenin. Scale bar $25 \mu \mathrm{m}$. d The examination of expression of $\beta$ III-tubulin using immunofluorescence staining. Scale bar $50 \mu \mathrm{m}$. All values were reported as mean \pm SD. ${ }^{*} P<0.01$ versus DMSO group (Color figure online)

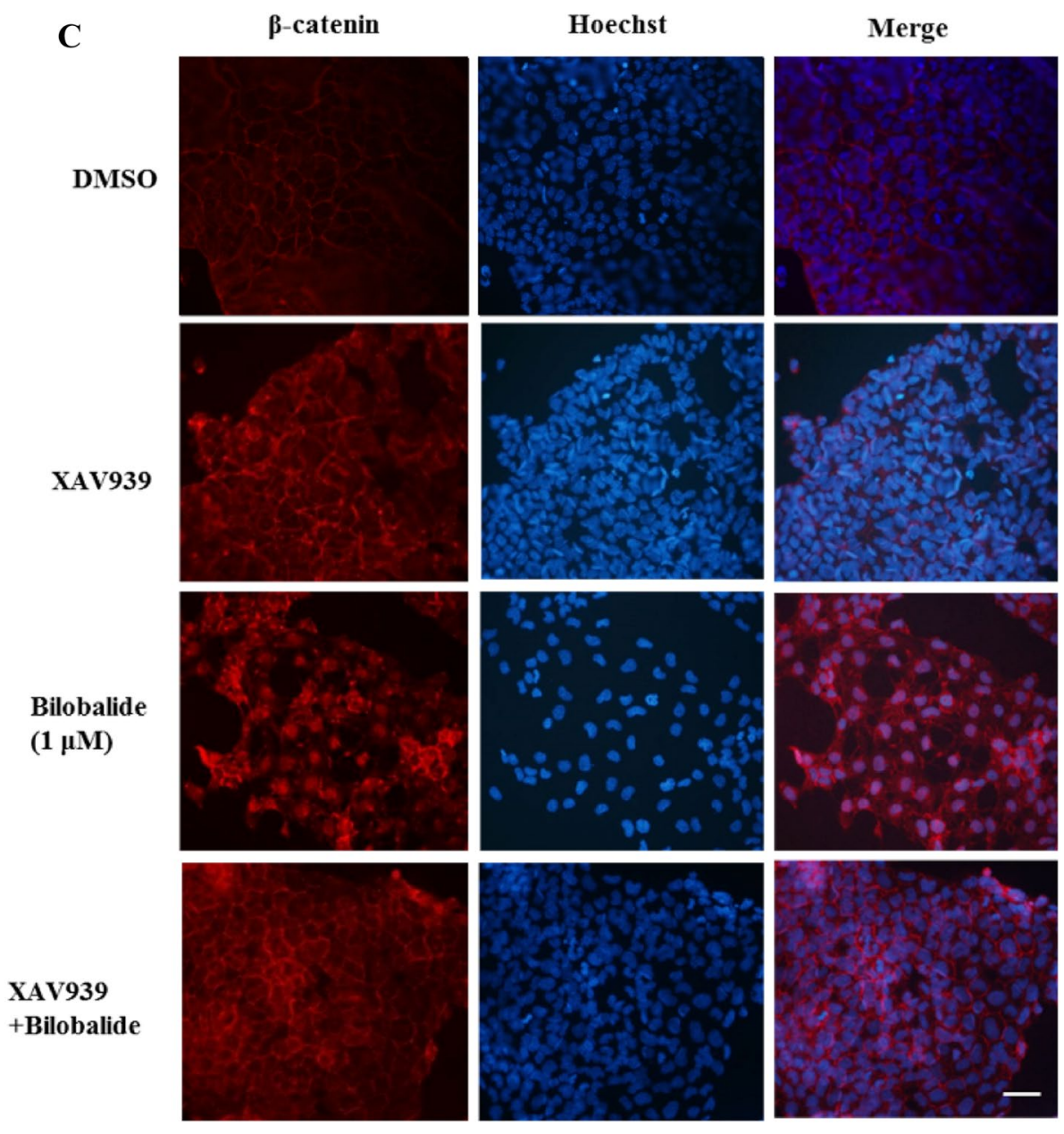

Publisher's Note Springer Nature remains neutral with regard to jurisdictional claims in published maps and institutional affiliations. 